\title{
Perturbations of the endocannabinoid system in mantle cell Iymphoma: correlations to clinical and pathological features
}

\author{
Agata M. Wasik ${ }^{1}$, Lina Nygren ${ }^{1}$, Stefan Almestrand ${ }^{1}$, Fang Zong ${ }^{1}$, Jenny Flygare ${ }^{2}$, \\ Stefanie Baumgartner Wennerholm ${ }^{3}$, Leonie Saft ${ }^{4}$, Patrik Andersson ${ }^{5}$, Eva Kimby ${ }^{3}$, \\ Björn E. Wahlin ${ }^{3}$, Birger Christensson ${ }^{1}$, and Birgitta Sander ${ }^{1}$ \\ ${ }^{1}$ Division of Pathology, Department of Laboratory Medicine, Karolinska Institutet, SE 14186 Stockholm, Sweden \\ ${ }^{2}$ Division of Clinical Chemistry, Department of Laboratory Medicine, Karolinska Institutet, SE 14186 Stockholm, Sweden \\ ${ }^{3}$ Division of Hematology, Department of Medicine, Karolinska Institutet, Karolinska University Hospital, SE 14186 Stockholm, \\ Sweden \\ ${ }^{4}$ Clinical Pathology, Karolinska University Hospital Solna, SE 17176 Stockholm, Sweden \\ ${ }^{5}$ Department of Hematology, Stockholm South Hospital, SE 11883 Stockholm, Sweden
}

Correspondence to: Agata M. Wasik, email: agata.wasik@ki.se

Keywords: mantle cell lymphoma, endocannabinoid system

Received: July 14, $2014 \quad$ Accepted: August 31, $2014 \quad$ Published: September 4, 2014

This is an open-access article distributed under the terms of the Creative Commons Attribution License, which permits unrestricted use, distribution, and reproduction in any medium, provided the original author and source are credited.

\section{Abstract}

The cannabinoid receptors are upregulated in many types of cancers, including mantle cell lymphoma (MCL) and have been suggested to constitute novel therapeutic targets. The expression pattern of the key members of the endocannabinoid system was analyzed in a well-characterized MCL patient cohort and correlated to biological features. 107 tumor tissues were analyzed for the mRNA levels of cannabinoid receptors 1 and 2 (CNR1 and CNR2) and the two main enzymes regulating the endocannabinoid anandamide levels in tissue: NAPEPLD and FAAH (participating in synthesis and degradation, respectively). NAPEPLD, CNR1 and CNR2 were overexpressed while FAAH expression was reduced in MCL compared to non-malignant B-cells. Both low CNR1 and high FAAH levels correlated with lymphocytosis $(p=0.016$ and $p=0.022$, respectively) and with leukocytosis $(p=0.0018$ and $p=0.047)$. Weak to moderate CNR1 levels were a feature of SOX11 negative MCL $(p=0.006)$. Both high CNR2 and high FAAH levels correlated to anemia $(p=0.0006$ and $p=0.038$, respectively). In conclusion, the relative expression of the anandamide synthesizing and metabolizing enzymes in MCL is heavily perturbed. This finding, together with high expression of cannabinoid receptors, could favor enhanced anandamide signaling and suggest that targeting the endocannabinoid system might be considered as part of lymphoma therapy.

\section{INTRODUCTION}

Overexpression of cyclin D1 is a key feature of mantle cell lymphoma (MCL). In the great majority of cases this is due to translocation of the CCND1 gene on chromosome 11 to the immunoglobulin locus on chromosome $14 \mathrm{t}(11 ; 14)(\mathrm{q} 13 ; \mathrm{q} 32)$ [1]. Recent studies have demonstrated that the lymphoma cells are highly dependent on signals from the microenvironment for their survival and proliferation [2]. Among crucial signaling pathways contributing to MCL pathogenesis are aberrant
BCR-signaling and alterations in PI3-kinase, WNT and TGF-beta signaling (reviewed in [3]). Promising new therapeutic agents such as inhibitors of PI3-kinase and Bruton's tyrosine kinase (BTK) interfere with such prosurvival signals $[3,4]$. Chemokine receptors are crucial for retaining MCL cells in close contact with stromal cells in the lymphoma niche and may constitute novel targets for therapy [5].

We and others have reported high expression of the G-protein coupled receptors cannabinoid receptor 1 and cannabinoid receptor 2 (encoded by CNR1 and 
CNR2, respectively) in MCL compared to non-malignant lymphoid tissue or purified non-malignant B-lymphocytes $[6,7]$. These receptors bind endogenous lipids, so called endocannabinoids. The cannabinoid receptors, the endocannabinoids and the enzymes regulating the levels of the endocannabinoids comprise the endocannabinoid system (ECS) [8]. Normally, CNR1 is hardly detected in lymphocytes but highly expressed in CNS and regulates synaptic signaling [9]. CNR2 is expressed in the immune system and the receptor protein regulates homing of B-lymphocytes and the architecture of B-cell areas in the spleen $[10,11]$. Furthermore, cannabinoid receptor 2, and to lesser extent cannabinoid receptor 1, participate in immune regulation by providing inhibitory or stimulatory signals depending on receptor expression levels, ligand concentrations and cell type $[12,13]$. One of the major endocannabinoids is $\mathrm{N}$-arachidonoylethanolamine, also called anandamide. The main enzyme responsible for the biosynthesis of anandamide is N-acyl phosphatidylethanolamine phospholipase D (NAPEPLD), while fatty acid amide hydrolase (FAAH) is the main enzyme metabolizing anandamide. Thus these two enzymes are key components in regulating the cellular anandamide levels.

Important functions of the cannabinoid receptors and endocannabinoid signaling have been described in several types of cancer (astrocytoma, glioma, breast-, prostate-, colon-, pancreatic - and hepatocellular cancer and also non-Hodgkin lymphoma) [13-15]. In general, cancer tissues express higher levels of cannabinoid receptors than the non-malignant counterparts and the endocannabinoid system is therefore considered as a potential novel therapeutic target in cancer therapy (reviewed in [14, 15]).

We have previously shown that exposure of MCL cells to cannabinoids induces cell death in vitro $[16,17]$ and reduces tumor growth in xenograft mouse models [18]. However, hitherto the clinical and biological impact of CNR1 and CNR2 expression in MCL has not been described.

In this study we investigated the expression of CNR1 and CNR2 and the major enzymes involved in the synthesis (NAPEPLD) and metabolism (FAAH) of the endocannabinoid anandamide in a well characterized cohort of MCL patients. The results are correlated to clinical and pathological features.

Table 1: Clinical and pathological features of the MCL patients included in the study.

\begin{tabular}{|c|c|}
\hline Clinical and pathological features & All, $n=107$ \\
\hline Median age (range) & $69.2(32.1-91.7)$ \\
\hline Age $>65(\%)$ & 63/107 (58.9) \\
\hline Sex, male/female & $75 / 32$ \\
\hline B symptoms $(\%)$ & $30 / 101(29.7)$ \\
\hline $\mathrm{ECOG}>=2(\%)$ & $5 / 102(4.9)$ \\
\hline Nodal presentation > 4 nodal sites $(\%)$ & $64 / 102(62.7)$ \\
\hline Splenomegaly (\%) & $44 / 96(45.8)$ \\
\hline Ann Arbor IV (\%) & $82 / 103(79.6)$ \\
\hline $\mathrm{WBC}>10 \times 10^{9} / \mathrm{L}(\%)$ & $26 / 102(25.5)$ \\
\hline Lymphocytes $>5 \times 10^{9} / \mathrm{L}(\%)$ (leukemic disease) & 20/101 (19.8) \\
\hline High serum LDH (\%) & $42 / 100(42)$ \\
\hline MIPI high risk (\%) & $28 / 79(35.4)$ \\
\hline Ki67 high $>=30 \%(\%)$ & $41 / 87(52.6)$ \\
\hline Anemia $(\mathrm{Hb}<120 \mathrm{~g} / \mathrm{L})$ & $30 / 103(29.1)$ \\
\hline SOX11 positivity (\%) & $78 / 85(91.8)$ \\
\hline p53 positivity $>20 \%$ of cells by IHC (\%) & $11 / 83(13.3)$ \\
\hline ASCT first line treatment $(\%)$ & $26 / 96(27.1)$ \\
\hline Blastoid morphology (\%) & $14 / 90(15.6)$ \\
\hline Indolent disease $(\%)$ & $14 / 92(15.2)$ \\
\hline Median OS y & 4.0 \\
\hline
\end{tabular}




\section{RESULTS}

\section{Clinical and pathological features of the MCL cases included}

In this study we analyzed the various components of the ECS in MCL diagnostic samples $(n=100)$ and relapse samples $(n=7)$ belonging to the well characterized population-based Stockholm cohort [19]. Lymph node biopsies constituted 81/107 samples. Clinical and pathological features of this cohort are presented in Table 1.

\section{Characterization of the endocannabinoid system involved in anandamide signaling in primary MCL samples}

Previously, we and others showed that the CNR1 and CNR2 are highly expressed in MCL compared to reactive lymphoid tissues $[6,17]$ or isolated non-malignant B-cell subpopulations [7]. In the current study, expression of CNR1, CNR2, NAPEPLD and FAAH was measured by PCR and results were normalized to the expression levels in non-malignant B-lymphocytes isolated from peripheral blood to map the expression pattern. Furthermore, we investigated whether the normalized expression values correlated to clinico-pathological data. Expression of NAPEPLD was analyzed by RT-PCR since the expression levels in non-malignant $\mathrm{B}$ cells were undetectable and could not be used for normalization. RT-PCR results showed that NAPEPLD was highly expressed in 18/18 $(100 \%)$ analyzed MCL cases (Figure 1a) but absent in normal B-cells. All other components of the ECS were analyzed by qPCR. In order to analyze the variation in mRNA expression levels and to rank the expression among cases we normalized to one of the peripheral blood samples, purified B-cells from buffy coat 1 . The values are expressed as relative fold increase (RFI). Thirteen MCL cases expressed higher FAAH than the control B-cells.

a)

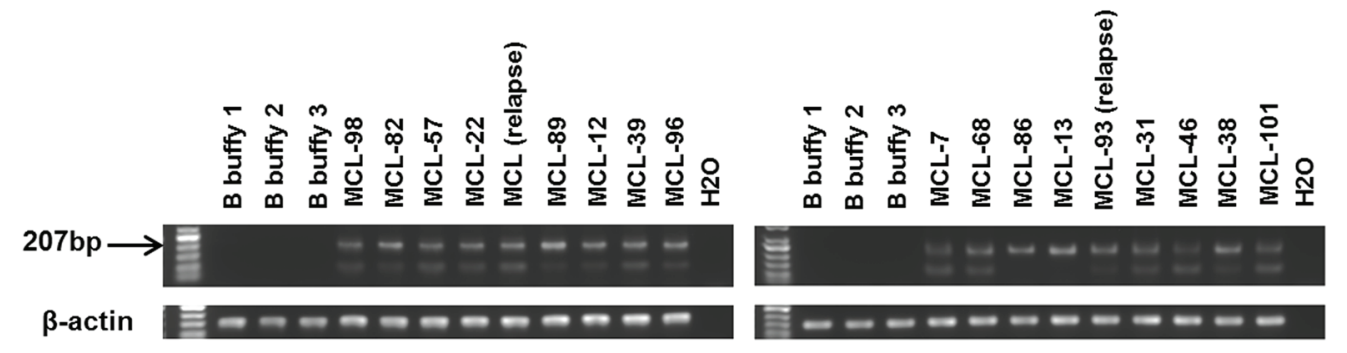

b)

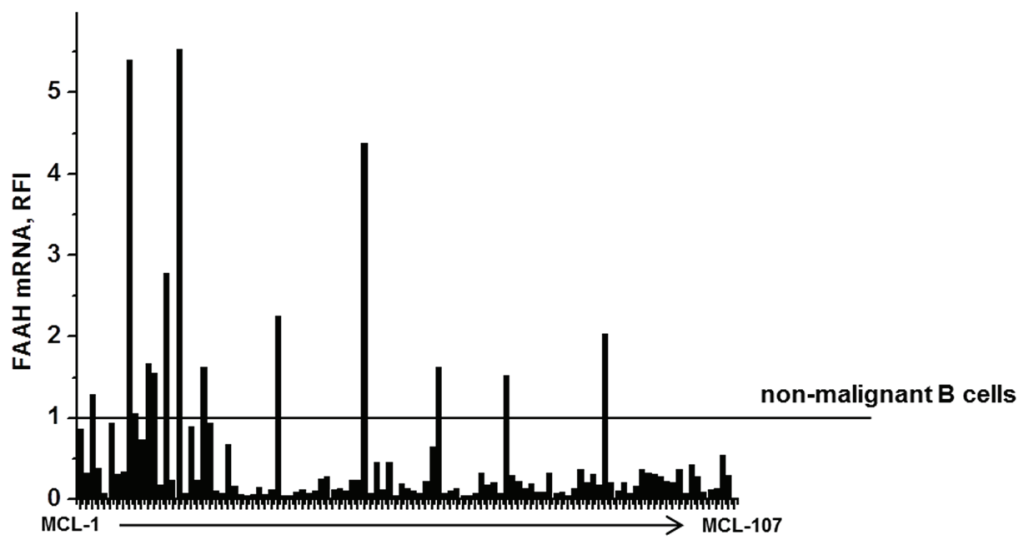

c)

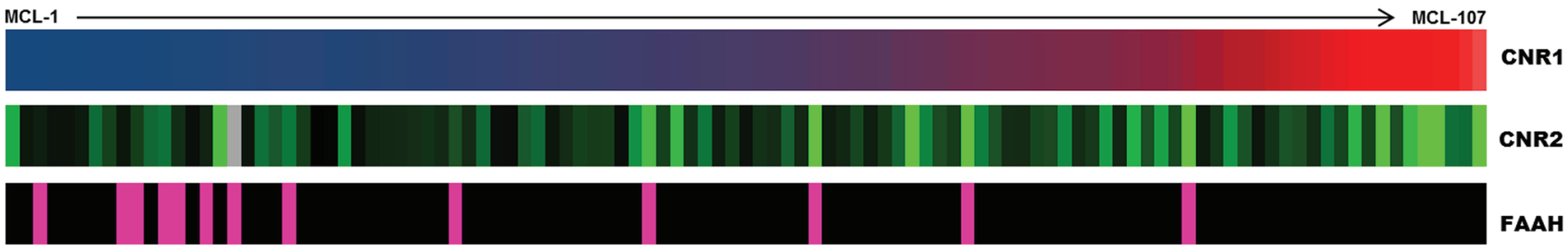

Figure 1: mRNA expression a) NAPEPLD in non-malignant B-cells and MCL analyzed by RT-PCR; b) FAAH mRNA in MCL analyzed by qPCR and expressed as relative fold increase (RFI) compared to non-malignant B cells; c) expression of CNR1, CNR2 and FAAH in MCL. Cases are sorted according to increasing levels of CNR1 (navy blue represents the lowest expression, $\mathrm{RFI}=0.63$, red corresponds to the highest expression, $\mathrm{RFI}=4652.37$ ). For CNR2 black shows the lowest expression $\mathrm{RFI}=1.06$, light green - highest $\mathrm{RFI}=176.97$, grey - data unknown). Cases with FAAH expression higher than in non-malignant B cells from buffy coat $1(\mathrm{RFI}>1)$ are marked in pink. 
Interestingly, in 95/107 (88\%) the expression of FAAH was low (Figure 1b). CNR1 mRNA was overexpressed in 105/107 (98\%) MCL samples but with a wide RFI range (0.63 to 4652.37) (Figure 1c). CNR2 mRNA was in all patients samples expressed at higher levels than in normal B-cells (RFI range 1,06 to 176,97) (Figure 1c). Expression levels of CNR1 and CNR2 correlated moderately (Spearman's correlation coefficient 0.39, $\mathrm{p}=0.00005$ ). Of note, in samples with high FAAH expression (12\%) the CNR1 expression was significantly lower $(p=0.003)$. The expression levels of CNR1, CNR2 and FAAH did not correlate to the tumor cell content in the tissue as measured by flow cytometry.

\section{CNR1, CNR2 and FAAH expression in relation to clinical and pathological features}

We normalized CNR1, CNR2 and FAAH expression to non-malignant B-cells from a single donor as described above and analyzed the expression levels in relation to clinical features (age, sex, anemia, thrombocytopenia, leukocytosis and lymphocytosis, LDH-levels, Ann Arbor stage, B-symptoms, splenomegaly, nodal presentation, mantle cell lymphoma international prognostic index (MIPI)), to overall survival (OS) and to pathological features (blastoid morphology, proliferation measured as Ki67-index, SOX11 positivity and p53 expression analyzed by IHC).

OS was analyzed in 100 of the cases with ECS expression data in the diagnostic biopsy. For assessing a possible association between gene expression and OS the $100 \mathrm{MCL}$ cases were grouped into two or three groups based on the CNR1, CNR2 or FAAH medians or tertiles of RFI values. Kaplan-Meier analysis showed no significant difference in OS with respect to CNR1, CNR2 nor FAAH expression.

Low CNR1 mRNA expression correlated to lymphocytosis defined as lymphocyte count $>5 \times 10^{9} / \mathrm{L}$ $(\mathrm{p}=0.016)$ (Figure 2a) and to leukocytosis $(\mathrm{p}=0.0018)$. In our cohort 84/90 cases were SOX11 positive by IHC. High CNR1 mRNA content was positively correlated to SOX11 positivity ( $\mathrm{p}=0.006$ ) (Figure $2 \mathrm{~b}$ ). We did not find any significant difference between CNR1 expression and other investigated features listed in Table 1.

We found a significant association between high CNR2 expression and anemia $(p=0.0006)$ (Figure 3$)$ but not with any of the other clinical and pathological features. High FAAH expression was significantly correlated to lymphocytosis $(p=0.022)$ and leukocytosis $(p=0.047)$, to anemia $(p=0.038)$ and to immunohistochemical $p 53$ positivity $(\mathrm{p}=0.001)$.

\section{DISCUSSION}

The ECS has attracted attention as a potential target for therapy in inflammatory disorders and cancer. A number of agents targeting various components of the ECS have been designed and tested in clinical trials $[13,15]$. MCL is an aggressive disease with usually poor survival [20] and new therapeutic options are clearly needed. We here analyzed the key components of the ECS and correlated the findings to clinico-pathological characteristics of the patients. Our study shows that the ECS is heavily perturbed in MCL. NAPEPLD was highly expressed in MCL compared to non-malignant B-cells.
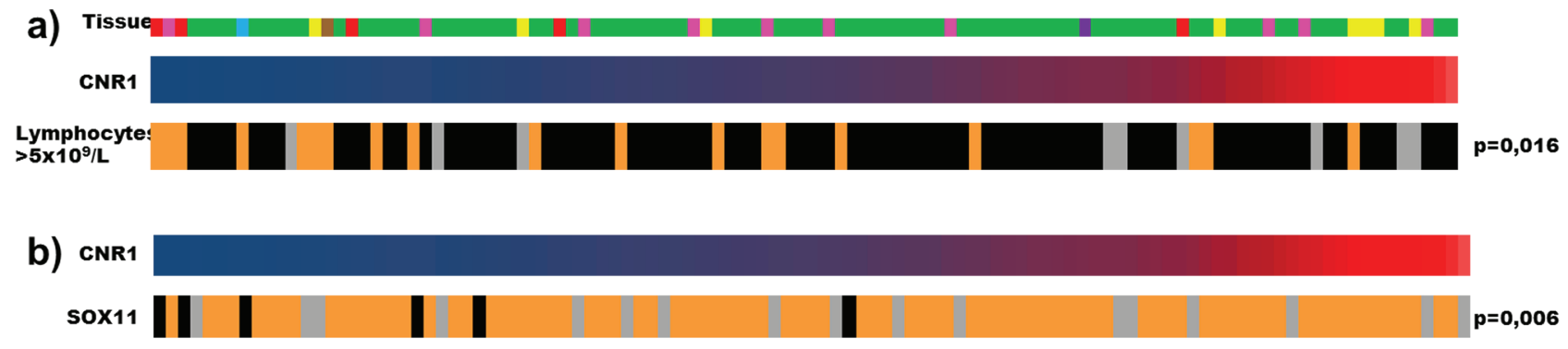

Figure 2: Graphical representation of statistical significance between CNR1 expression levels and other parameters. Tissue types in which gene expression was investigated are color-coded: green - lymph node, pink - tonsil, yellow - spleen, brown - bone marrow, purple - gastrointestinal tract, red - blood, blue - pleura. MCL cases were sorted according to increasing levels of CNR1 expression (navy blue shows the lowest expression $\mathrm{RFI}=0.63$, red - the highest $\mathrm{RFI}=4652.37$ ): a) low levels of CNR1 mRNA correlate to lymphocytosis (lymphocytes $>5 \times 10^{9} / \mathrm{L}$ ), marked in orange, grey color - data unknown; b) CNR1 mRNA levels correlate to immunoreactivity for SOX11. SOX11 positive cases are marked in orange, SOX11 negative in black, grey color - data unknown.

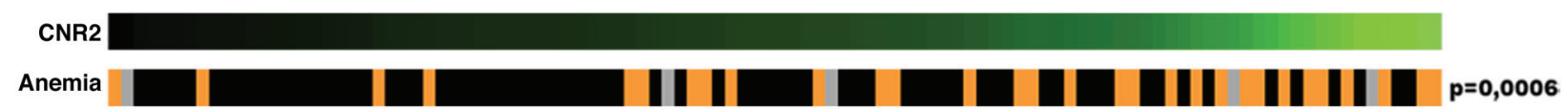

Figure 3: Graphical representation of the statistical significance between high CNR2 levels (blue - lowest expression, RFI=1.06, red - highest RFI=176.97) and anemia (orange color - anemia, grey color - data unknown). 
88\% MCL cases investigated had lower FAAH mRNA levels compared to non-malignant B-cells, suggesting that the malignant cells might accumulate anandamide. In addition, MCL expressed high levels of both cannabinoid receptors, favoring endocannabinoid signaling.

A subset of cases was characterized by high FAAH and weak to moderate CNR1 expression, suggesting that these cases do not have an upregulated endocannabinoid system and may have a different pathophysiology. In line with this both high FAAH and low CNR1 in tumor tissue were seen in patients with lymphocytosis at diagnosis.

Recently, the transcription factor SOX11 was described to be aberrantly expressed in approximately $95 \%$ of MCL cases, while normal B cells are SOX11 negative [19, 21-24]. Our study contained mostly SOX11 positive cases, and we found a significant correlation between high SOX11 expression and high expression of CNR1. Among the SOX11 negative MCL 5/6 cases were in the lowest range (lowest 25\%) of CNR1 expression. Interestingly, Fernandez et al. described a subset of nonnodal leukemic MCL cases with indolent clinical course, characterized by low SOX11 expression [21]. In their gene expression analysis of SOX11 negative and SOX11 positive MCL, CNR1 was identified as significantly lower in SOX11 negative MCL. In our cohort, we had only 1 case that would correspond to the leukemic, indolent MCL described by Fernandez et al. [21] and in fact, in our study leukemic disease was associated with nodal presentation and correlated to poorer overall survival $(p=0.037)$. In spite of this difference, low CNR1 expression seems to be a feature of lymphocytosis in MCL. MCL survival and proliferation depend on the signals from the tumor microenvironment [2]. Importantly, MCL cells disrupted from the lymphoid/bone marrow microenvironment lose pro-survival support from stromal cells $[2,25]$. Our data indicate that lymphocytosis in MCL is characterized by low CNR1 expression, implying that high CNR1 is associated with homing to lymphoid tissue, increased adhesion and/or reduced egress of MCL cells from lymph nodes. Signaling through CNR1 might thus be involved in regulating the retention of MCL cells in lymphoid tissue a niche that provides optimal conditions for tumor cell growth and survival and potentially enhanced resistance to many therapeutic agents. The possible involvement of CNR1 in leukocyte homing is supported by several experimental studies in which CNR1 stimulation altered adhesive properties. Kianian et al. showed recently that CNR1 inhibition by specific antagonist AM281 reduced leukocyte recruitment within the intestinal microvasculature in experimental endotoxemia in Lewis rats [26]. CNR1 has further been suggested to be involved in adhesion of T cells [27] and synovial fibroblasts [28] in experimental disease models.

We found that all MCL cases expressed higher levels of CNR2 than non-malignant B-cells. A significant correlation between high CNR2 expression and anemia was found. As the cannabinoid receptor 2 is responsible for retention of immature B cells in the bone marrow [29] and leukemic cells in the bone marrow disrupt the normal hematopoiesis [30], we hypothesized that the anemia might be secondary to high bone marrow infiltration but morphological re-evaluation of staging bone marrow biopsies did not support this hypothesis (data not shown).

In summary, this study investigated the expression of cannabinoid receptors and enzymes regulating levels of the endocannabinoid anandamide in diagnostic samples of MCL. MCL has high expression of a key enzyme in the anandamide synthesis, NAPEPLD and mostly low expression of the anandamide metabolizing enzyme FAAH, suggesting that high anandamide levels are favored in this lymphoma subtype. In addition, the expression of both cannabinoid receptors was upregulated in most cases as compared to non-malignant B-cells. In contrast, only a few cases were characterized by low CNR1 expression and high expression of FAAH and these MCL had elevated lymphocyte count at diagnosis. Our data point to a role for the ECS in homing, adhesion and egress of MCL cells from the lymphoid tissue. Importantly, the ECS is druggable and ligands to CB1 and CB2 are used in clinical trials for various diseases including multiple sclerosis and cancer [15, 31-33]. This suggests that targeting the endocannabinoid system might be a possible novel treatment modality in MCL.

\section{MATERIALS AND METHODS}

\section{Patients and clinical data}

Diagnostic samples with available frozen tissue from patients diagnosed with MCL between January 1994 and March 2013 at the Pathology Department of Karolinska University Hospital were retrieved. In total, $107 \mathrm{MCL}$ cases were identified and 100 of these were samples taken at diagnosis, before start of treatment. These patients are part of a heterogeneously treated, unselected, populationbased cohort [19]. Baseline and follow-up clinical data were obtained from hospital files.

\section{Tumor tissue, and immunohistochemical analysis}

Tumor tissue from lymph nodes $(n=81)$, spleens $(n=8)$, tonsils $(n=10)$ or gastrointestinal biopsies $(n=1)$ removed for diagnostic purposes were snap frozen and stored at $-80^{\circ} \mathrm{C}$ until use. In some instances cell suspensions from bone marrow $(n=1)$, blood $(n=5)$ or pleural fluid $(n=1)$ were retrieved by Ficoll separation (Ficoll-Paque PLUS, GE Healthcare), viability frozen in DMSO and stored at $-150^{\circ} \mathrm{C}$.

Lymphomas were diagnosed according to the WHO 
classification [1]. All cases were cyclin D1 positive by immunohistochemistry (IHC) and/or positive for $\mathrm{t}(11 ; 14)$ (q13;q32) translocation by interphase FISH or cytogenetic analysis. IHC staining for cyclin D1, Ki-67, p53 and SOX11 was done on whole sections of paraffin embedded diagnostic biopsies as previously described [19].

\section{RNA isolation and cDNA synthesis}

RNA was isolated using the RNeasy Plus mini kit (Qiagen) according to the manufacturer's protocol. Quantification and quality of the RNA preparations were measured using the NanoDrop ND-1000 spectrophotometer (Saveen Werner). Complementary DNA (cDNA) was synthesized using the Omniscript Reverse Transcription (RT) kit (Qiagen) according to manufacturer's protocol. RNaseOut Recombinant Ribonuclease inhibitor and the Oligo dT primers used were purchased from Invitrogen.

\section{Quantitative PCR (qPCR)}

mRNA expression levels of CNR1, CNR2, NAPEPLD and FAAH were assessed by Real-Time PCR using Platinum SYBR Green qPCR SupermixUDG (Invitrogen) according to the manufacturer's protocol. Primers sequence (Eurogentec) for the selected genes were as follows: CNR1 forward: 5-CATTAAGACGGTGTTTGCATTCT-3, reverse: 5-CGTGTCGCAGGTCCTTACTC-3; CNR2 forward: 5 - GACACGGACCCCTTTTTGCT-3, reverse 5 - CCTCGTGGCCCTACCTATCC-3; NAPEPLD forward: 5-CGGAGCCTGCATCTCTGAAG-3, reverse: 5-AGGATATTGGCTGCTTGTCATCA-3; FAAH forward: 5-GAAGTCTCGTTCGGCTGGAA-3, reverse: 5-ACTGGGCAATCACGGTTTTG-3; $\beta$-actin forward: 5-AAAGACCTGTACGCCAACACA-3, reverse: 5-AGTACTTGCGCTCAGGAGGA-3. Each sample was prepared in triplicates in a 96 well plate (BioRad) and the reactions were performed with the $\mathrm{C} 1000$ Thermal cycler (BioRad). An initial step was performed at $95^{\circ} \mathrm{C}$ for 2 min, followed by 40 cycles of $95^{\circ} \mathrm{C}$ for 15 seconds and finished by $57^{\circ} \mathrm{C}$ for $30 \mathrm{sec}$. The results were analyzed and cycle threshold $(\mathrm{Ct})$ values of transcripts were quantified using CFX manager software (BioRad). The $\Delta \mathrm{Ct}$ values were calculated using $\beta$-actin as reference. Expression levels of CNR1, CNR2 and FAAH of mRNA were normalized to the expression levels of the respective gene in the B-cells isolated from the buffy coat 1 (used as a standard). NAPEPLD mRNA in B-cells from buffy coats from three different healthy donors was undetectable and thus the PCR amplification products for NAPEPLD were run on the $1.5 \%$ agarose (Gibco) gel. The image was taken using FluorChem SP (Alpha Innotech). Two bands of the NAPEPLD PCR product were cut out from the gel, DNA was extracted using kit from Fermentas, DNA was precipitated using Pellet Paint NF Co-precipitant from Novagene and sent off for sequencing to KIGene to confirm that the band at 207bp represented NAPEPLD.

\section{Statistical analysis}

Overall survival (OS) was calculated from the date of MCL diagnosis to the date of death. Associations with survival were evaluated using Kaplan-Meier curves and the log-rank test. Statistical analysis included Spearman rank correlation and Mann-Whitney test. A p value of $<0.05$ was considered significant.

\section{Ethical permission}

The Regional Central Ethical Review Board at Karolinska Institutet has approved the research and given all necessary ethical permissions.

\section{ACKNOWLEDGEMENTS}

Authors thank Åsa Jeppsson Ahlberg and Mariann Wikman and other personnel at the Flow Cytometry Lab at Karolinska University Huddinge. The project was supported by The Swedish Cancer Society, The Swedish Research Council, The Cancer Society in Stockholm, The Karolinska Institutet Funds and The Stockholm County Council.

\section{REFERENCES}

1. Swerdlow S, Campo E, Harris NE, Jaffe ES, Pileri SA, Stein H, Thiele J, Vardiman JW. WHO Classification of Tumours of Haematopoietic and Lymphoid Tissues. IARC press, Lyon. 2008.

2. Burger JA, Ford RJ. The microenvironment in mantle cell lymphoma: cellular and molecular pathways and emerging targeted therapies. Semin Cancer Biol. 2011; 21:308-312.

3. Parekh S, Weniger MA, Wiestner A. New molecular targets in mantle cell lymphoma. Semin Cancer Biol. 2011; 21: 335-346.

4. Chang BY, Francesco M, De Rooij MF, Magadala P, Steggerda SM, Huang MM, Kuil A, Herman SE, Chang S, Pals ST, Wilson W, Wiestner A, Spaargaren M, Buggy JJ, Elias L. Egress of CD19(+)CD5(+) cells into peripheral blood following treatment with the Bruton tyrosine kinase inhibitor ibrutinib in mantle cell lymphoma patients. Blood. 2013; 122: 2412-2424.

5. Kurtova AV, Tamayo AT, Ford RJ, Burger JA. Mantle cell lymphoma cells express high levels of CXCR4, CXCR5, and VLA-4 (CD49d): importance for interactions with the stromal microenvironment and specific targeting. Blood. 2009; 113: 4604-4613. 
6. Islam TC, Asplund AC, Lindvall JM, Nygren L, Liden J, Kimby E, Christensson B, Smith CI, Sander B. High level of cannabinoid receptor 1 , absence of regulator of $\mathrm{G}$ protein signalling 13 and differential expression of Cyclin D1 in mantle cell lymphoma. Leukemia. 2003; 17: 1880-1890.

7. Ek S, Hogerkorp CM, Dictor M, Ehinger M, Borrebaeck CA. Mantle cell lymphomas express a distinct genetic signature affecting lymphocyte trafficking and growth regulation as compared with subpopulations of normal human B cells. Cancer Res. 2002; 62: 4398-4405.

8. Galve-Roperh I, Chiurchiu V, Diaz-Alonso J, Bari M, Guzman M, Maccarrone M. Cannabinoid receptor signaling in progenitor/stem cell proliferation and differentiation. Prog Lipid Res. 2013; 52: 633-650.

9. De Petrocellis L, Di Marzo V. An introduction to the endocannabinoid system: from the early to the latest concepts. Best Pract Res Clin Endocrinol Metab. 2009; 23: 1-15.

10. Muppidi JR, Arnon TI, Bronevetsky Y, Veerapen N, Tanaka M, Besra GS, Cyster JG. Cannabinoid receptor 2 positions and retains marginal zone $\mathrm{B}$ cells within the splenic marginal zone. J Exp Med. 2011; 208: 1941-1948.

11. Basu S, Ray A, Dittel BN. Cannabinoid receptor 2 is critical for the homing and retention of marginal zone $\mathrm{B}$ lineage cells and for efficient T-independent immune responses. $\mathrm{J}$ Immunol. 2011; 187: 5720-5732.

12. Basu S, Dittel BN. Unraveling the complexities of cannabinoid receptor 2 (CB2) immune regulation in health and disease. Immunologic Res. 2011; 51: 26-38.

13. Wasik AM, Christensson B, Sander B. The role of cannabinoid receptors and the endocannabinoid system in mantle cell lymphoma and other non-Hodgkin lymphomas. Semin Cancer Biol. 2011; 21: 313-321.

14. Pisanti S, Picardi P, D’Alessandro A, Laezza C, Bifulco M. The endocannabinoid signaling system in cancer. Trends Pharmacol Sci. 2013; 34: 273-282.

15. Velasco G, Sanchez C, Guzman M. Towards the use of cannabinoids as antitumour agents. Nat Rev Cancer. 2012; 12: 436-444.

16. Wasik AM, Almestrand S, Wang X, Hultenby K, Dackland AL, Andersson P, Kimby E, Christensson B, Sander B. WIN55,212-2 induces cytoplasmic vacuolation in apoptosis-resistant MCL cells. Cell Death Dis. 2011; 2: e225.

17. Gustafsson K, Wang X, Severa D, Eriksson M, Kimby E, Merup M, Christensson B, Flygare J, Sander B. Expression of cannabinoid receptors type 1 and type 2 in non-Hodgkin lymphoma: growth inhibition by receptor activation. Int $\mathrm{J}$ Cancer. 2008; 123: 1025-1033.

18. Gustafsson K, Christensson B, Sander B, Flygare J. Cannabinoid receptor-mediated apoptosis induced by $\mathrm{R}(+)$-methanandamide and Win55,212-2 is associated with ceramide accumulation and $\mathrm{p} 38$ activation in mantle cell lymphoma. Mol Pharmacol. 2006; 70: 1612-1620.
19. Nygren L, Baumgartner Wennerholm S, Klimkowska M, Christensson B, Kimby E, Sander B. Prognostic role of SOX11 in a population-based cohort of mantle cell lymphoma. Blood. 2012; 119: 4215-4223.

20. Perez-Galan P, Dreyling M, Wiestner A. Mantle cell lymphoma: biology, pathogenesis, and the molecular basis of treatment in the genomic era. Blood. 2011; 117: 26-38.

21. Fernandez V, Salamero O, Espinet B, Sole F, Royo C, Navarro A, Camacho F, Bea S, Hartmann E, Amador V, Hernandez L, Agostinelli C, Sargent RL, Rozman M, Aymerich M, Colomer D, et al. Genomic and gene expression profiling defines indolent forms of mantle cell lymphoma. Cancer Res. 2010; 70: 1408-1418.

22. Meggendorfer M, Kern W, Haferlach C, Haferlach T, Schnittger S. SOX11 overexpression is a specific marker for mantle cell lymphoma and correlates with $\mathrm{t}(11 ; 14)$ translocation, CCND1 expression and an adverse prognosis. Leukemia. 2013; 27: 2388-2391.

23. Ek S, Dictor M, Jerkeman M, Jirstrom K, Borrebaeck CA. Nuclear expression of the non B-cell lineage Sox11 transcription factor identifies mantle cell lymphoma. Blood. 2008; 111: 800-805.

24. Wang X, Asplund AC, Porwit A, Flygare J, Smith CI, Christensson B, Sander B. The subcellular Sox11 distribution pattern identifies subsets of mantle cell lymphoma: correlation to overall survival. Br J Haematol. 2008; 143: 248-252.

25. Medina DJ, Goodell L, Glod J, Gelinas C, Rabson AB, Strair RK. Mesenchymal stromal cells protect mantle cell lymphoma cells from spontaneous and drug-induced apoptosis through secretion of B-cell activating factor and activation of the canonical and non-canonical nuclear factor kappaB pathways. Haematologica. 2012; 97: 1255-1263.

26. Kianian M, Kelly ME, Zhou J, Hung O, Cerny V, Rowden G, Lehmann C. Cannabinoid receptor 1 inhibition improves the intestinal microcirculation. Clin Hemorheol Microcirc. 2013.

27. Rossi B, Zenaro E, Angiari S, Ottoboni L, Bach S, Piccio L, Pietronigro EC, Scarpini E, Fusco M, Leon A, Constantin $\mathrm{G}$. Inverse agonism of cannabinoid CB1 receptor blocks the adhesion of encephalitogenic $\mathrm{T}$ cells in inflamed brain venules by a protein kinase A-dependent mechanism. J Neuroimmunol. 2011; 233: 97-105.

28. Lowin T, Zhu W, Dettmer-Wilde K, Straub RH. Cortisolmediated adhesion of synovial fibroblasts is dependent on the degradation of anandamide and activation of the endocannabinoid system. Arthritis and rheumatism. 2012; 64: 3867-3876.

29. Pereira JP, An J, Xu Y, Huang Y, Cyster JG. Cannabinoid receptor 2 mediates the retention of immature $\mathrm{B}$ cells in bone marrow sinusoids. Nat Immunol. 2009; 10: 403-411.

30. Colmone A, Amorim M, Pontier AL, Wang S, Jablonski E, Sipkins DA. Leukemic cells create bone marrow niches that disrupt the behavior of normal hematopoietic progenitor cells. Science. 2008; 322: 1861-1865. 
31. Guzman M, Duarte MJ, Blazquez C, Ravina J, Rosa MC, Galve-Roperh I, Sanchez C, Velasco G, Gonzalez-Feria L. A pilot clinical study of Delta9-tetrahydrocannabinol in patients with recurrent glioblastoma multiforme. Br J Cancer. 2006; 95: 197-203.

32. Fowler CJ, Gustafsson SB, Chung SC, Persson E, Jacobsson SO, Bergh A. Targeting the endocannabinoid system for the treatment of cancer-a practical view. Curr Top Med Chem. 2010; 10: 814-827.

33. Flygare J, Sander B. The endocannabinoid system in cancerpotential therapeutic target? Semin Cancer Biol. 2008; 18: 176-189. 